\title{
El Liber de anima rationali, ¿primera obra antiaverroísta de Ramon Llull?
}

\author{
Celia López Alcalde \\ Universidad Autònoma de Barcelona \\ Celia.lopez@uab.es
}

Resumen: Este artículo tiene como propósito el dar a conocer alguna de las problemáticas que han surgido en la edición y estudio que estamos llevando a cabo de la obra conocida como Liber de anima rationali $(L A R)$ de Ramon Llull. Para ello, en primer lugar esbozaremos en pocos trazos aspectos generales de la obra, incluyendo un repaso por la bibliografía específica generada y la exposición de nuevos elementos emergentes que ha proporcionado el estudio desempeñado hasta ahora, así como también el planteamiento de cuestiones aún por resolver. Tras esta introducción concentraremos nuestro interés en un elemento inédito que aparece en el libro y en torno al cual gira la atención de este artículo, a saber, la referencia a Averroes y a lo que se ha llamado su doctrina del monopsiquismo. En este apartado trataremos de contextualizar la referencia dentro del pensamiento luliano y relacionarla con otras obras del autor consideradas antiaverroístas en las que se combate dicha doctrina, para ver qué significa esta referencia en la historia de la campaña de Llull contra el llamado averroísmo. Por último, la conclusión nos llevará a plantear la necesidad de revisar el lugar del $L A R$ dentro de la trayectoria productiva de Ramon Llull.

Palabras clave: Anti-Averroísmo, Aristoteles, Averroes (Ibn Rušd), De anima, Averroísmo Latino, Liber de anima rationali, Monopsiquismo, Ramon Llull.

Was the Liber de anima rationali the first anti-averroistic work by Ramon Llull?

\begin{abstract}
This paper presents some of the issues that have emerged during the preparation of a critical edition and study of Ramon Llull's Liber de anima rationali $(L A R)$. First some general characteristics of the work will be discussed, including a survey of relevant bibliography, some conclusions reached while preparing the edition, and finally setting out some problems that still need to be solved. Following this, we will concentrate on an unnoticed aspect of this work, a reference to Averroes and to monopsychism, contextualizing it within Lullian thought and relating it to other anti-averroistic works where Llull refutes this doctrine. This will allow us to appreciate what the implications of the
\end{abstract}


reference are in the development of Llull's crusade against Averroism, leading us to re-examine the position of the $L A R$ within Ramon Llull's literary production.

Key words: Anti-Averroism, Aristotle, Averroes (Ibn Rušd), De anima, Latin Averroism, Liber de anima rationali, Monopsychism, Ramon Llull

\section{INTRODUCCIÓN}

\subsection{El LAR. Estudios sobre la obra}

La producción de Ramon Llull abarca un variado panorama de ámbitos, entre los cuales se encuentra el discurso filosófico en sentido estricto, desarrollado en un gran número de obras. Entre esta producción figura el Liber de anima rationali, obra que trata de exponer y delimitar las características esenciales y las funciones del alma racional, un campo de análisis que inagura, como tantos otros, Aristóteles, con su tratado Sobre el alma ${ }^{1}$. Ya Éphrem Longpré en el Dictionnaire de théologie catholique ${ }^{2}$ se refirió a la obra como «un intéressant traité de psychologie». Como afirmara en su día Walter Artus, la obra constituye cierta unidad temática con el Liber de homine, ambos tratados de carácter antropológico ${ }^{3}$.

Robert Pring-Mill, en su descripción de la cosmovisión luliana ${ }^{4}$ hace referencia a la obra y resalta el carácter trinitario que enlaza directa o indirectamente con la visión agustiniana: el alma humana, con sus tres facultades, la memoria, el intelecto y la voluntad, es «imagen de las relaciones de las tres Personas de la Trinidad $»^{5}$. Relacionado con este elemento trinitario, Jordi Gayà, en su estudio sobre los correlativos lulianos, habla de «la estructuración correlativa del alma humana» que Llull plasma en su libro sobre el alma racional. Jocelyn Hillgarth, por su parte, muestra cómo el discípulo de Llull, Thomas Le Myésier, en su Introductio in artem Remundi expone las doctrinas lulianas, entre ellas su visión del alma; también añade la obra al Electorium (1325) y la cita muy a menudo por considerarla una obra importante de la producción luliana ${ }^{7}$. Fernando

${ }^{1}$ El tratado Sobre el alma está traducido al latín ya en el siglo XII, por Jacobo de Venecia. El texto latino se encuentra editado junto al comentario de Alberto Magno en el tomo VII/1 de la Editio Coloniensis.

${ }^{2}$ Longpré, É., «Lulle, Raymond (Le bienheureux)», Dictionnaire de théologie catholique, vol. IX/1, Letouzey et Ané, Paris, 1926, cols. 1072-1141.

${ }^{3}$ En Artus, W., «Ramon Llull on the Pre-Eminence of Man in a God-Centered Universe», Antonianum, 66 (1991), pp. 140-154 (p. 141).

${ }^{4}$ Pring-Mill, R.D.F., El microcosmos lul-lià, Editorial Moll, Palma, 1961.

5 Ibidem, p. 130: «'ymagen' de les tres relacions entre les tres Persones de la Trinitat».

${ }^{6}$ Gayà, J., La teoría luliana de los correlativos. Historia de su formación conceptual, Impresos Lope, Palma, 1979, p. 118.

${ }^{7}$ Hillgarth, J.N., Ramon Lull and Lullism in Fourteenth-Century France, The Clarendon Press, Oxford, 1971, p. 224 y siguientes. 
Domínguez, en el inventario de las obras lulianas, comenta la importancia de la obra en cuanto a su explicación clarificadora del papel de las facultades del alma dentro del sistema luliano y en cuanto a la exposición que ofrece de la definitiva epistemología luliana ${ }^{8}$.

Centrándonos en su estructura, la obra, tal y como anuncia el prólogo, se divide en diez partes que corresponden a las cuestiones que son establecidas en la Tabula generalis de 1294, obra que presenta un sistema combinatorio de acercamiento metodológico ${ }^{9}$ las cuestiones de conocimiento:

...quapropter volumus de anima rationali sub compendio notitiam dare, de eius principiis naturalibus ac suis operationibus naturalibus atque moralibus; et erit suum scrutamen secundum regulas Tabulae generalis. ${ }^{10}$

Con esta sistematización, Llull pretende como fin último llegar a mostrar de qué manera se relaciona en el hombre la realidad espiritual y la material, y demostrar cómo el alma y sus potencias — recordar, entender y amar - tienen como fin último Dios.

Más allá del análisis interno de la obra, cuestiones de otra índole suscitan el interés en relación a su estudio. Una de ellas es, como en tantos otros casos de obras lulianas, la cuestión lingüística. Longpré ${ }^{11}$ constató la existencia de dos tradiciones lingüísticas: una en catalán, con la pervivencia de un manuscrito de finales del siglo XIV, principios del XV (Múnich, Bayerische Staatsbibliothek, Hisp. 52 (596)), y la otra con una importante cantidad de manuscritos del siglo $\mathrm{XV}$ y posteriores escritos en latín ${ }^{12}$.

Esta situación, por otra parte común en la producción luliana, convierte la edición en una cuestión compleja; como hiciera constar en su artículo sobre el problema de la edición de las obras lulianas, Alexander Fidora ${ }^{13}$, tras un primer análisis filológico, considera que se puede afirmar con cierta seguridad que la redacción catalana es fuente de la traducción latina. Las pruebas esgrimidas nos sirven como hipótesis de trabajo y según esto tenemos en cuenta dicha versión

${ }^{8}$ Domínguez, F., «Works», en A. Fidora y J. E. Rubio (eds.), Raimundus Lullus: An Introduction to his Life, Works and Thought (ROL - Supplementum Lullianum II), Brepols, Turnhout, 2008, pp. 125-242 (p. 171).

9 La Tabula generalis expone de manera sistemática la combinación de los Principios (Bonitas, Magnitudo, Aeternitas, etc.) y las Reglas (Possibilitas, Quidditas, Materialitas, etc.); el LAR se construye entorno a la aplicación de las reglas generadoras de preguntas y respuestas: utrum, quod, de quo, quare, quantum, quale, quando, ubi, quo modo, cum quo.

${ }_{10}$ Ms. Palma, Biblioteca Pública, 1007, f. 1ra.

${ }^{11}$ Longpré, «Lulle, Raymond...», col. 1093.

12 En total disponemos de 26 manuscritos (tres de ellos fragmentarios) y dos ediciones del texto latino, una de Alcalá de 1519, por Nicolás de Pax, y la de Maguncia de 1737 (= MOG VI), por Franz Philipp Wolff.

${ }^{13}$ Fidora, A., «La doble tradició de les obres lul-lianes i el problema de les edicions i traduccions modernes», en A. Fidora y E. Trenc (eds.), Segon col-loqui europeu d' estudis catalans, vol. 1: La recepció de la literatura catalana medieval a Europa, Éditions de la Tour Gile, Péronnas, 2007, pp. 5-15. 
en la edición crítica del texto latino que estamos realizando. Del estudio realizado hasta ahora se pueden vislumbrar tres subgrupos dentro de la versión latina, aunque ésta es una cuestión aún por resolver a través de la comparación de los distintos manuscritos ${ }^{14}$.

\section{2. ¿Liber de anima rationali o Liber novus de anima rationali? La cuestión del título}

Asimismo, el estudio filológico de la obra en vistas a la edición abre nuevas consideraciones y nuevos planteamientos que incumben incluso al propio título de la obra: Libro del alma racional, en su equivalente latino o catalán. Este título pone en relación la obra con el clásico de Aristóteles sobre el alma, De anima; Llull lo cita del siguiente modo en el listado de obras del Estagirita dado en la Doctrina pueril:

E lo libre De anima racional parla de la substancia d'anima e de la esperitualitat e incorupció, e de sos poders, e com endressa lo cors e com pren los objecs. E parla con es different a les alt[res a]nimes inracionals. E assó fa per saber la natura de la anima racional.» ${ }^{15}$

Llull, al nombrar su obra como hubo nombrado la de Aristóteles establece una relación manifiesta entre ambas ${ }^{16}$. Inscrito en la misma tradición, el libro tratará de ofrecer nuevas soluciones a la problemática psicológica a través de la aplicación de su Arte. La idea de novedad viene ratificada por el título que presentan la mayoría de los manuscritos, incluído el manuscrito catalán: en efecto, la edición catalana, que parte del manuscrito catalán de Múnich, se presenta como «novell Libre de ànima racional», y el epíteto de «novus, novum» se da asimismo en casi todos los manuscritos latinos.

Este hecho ha sido descuidado o no suficientemente apreciado por los estudios recientes ${ }^{17}$. Como expresa Michela Pereira en su artículo «Le 'nuove'

${ }^{14}$ Longpré («Lulle, Raymond...», col. 1093), habla de dos versiones latinas distintas: una que representarían el manuscrito de San Candido, Biblioteca della Collegiata, VIII, B. 8 y el manuscrito de Oxford, Merton College, 89; y la otra el resto de los manuscritos. Para nosotros esta doble agrupación presenta dificultades.

15 Ramon Llull, Doctrina pueril, edición crítica de Joan Santanach i Suñol (NEORL VII), Patronat Ramon Llull, Palma 2005, p. 203.

${ }^{16} \mathrm{La}$ incorporación por parte de Llull del adjetivo «racional» no es de hecho habitual ni acorde con la realidad que la obra presenta, ya que señala a uno de los tipos o facultades del alma (II 1, 413b 10) y no el alma en su totalidad, como en verdad la presenta Aristóteles en la obra, entendida como «entelequia primera de un cuerpo que en potencia tiene vida» (II 1, 412a 27-28).

17 En la valiosa base de datos del Centre de Documentació Ramon Llull, http://orbita.bib.ub.es/llull/index.asp, la forma «Novell libre de ànima racional» se registra como variante del título. Según lo comentado, creemos que en la edición y bibliografía debería incluirse «novell» como parte del título: Novell libre de ànima racional. En el listado de obras filosóficas de Llull de la Historia de la Filosofía española de los hermanos Carreras i Artau aparece de este modo; cf. Carreras y Artau, T. y J., Historia de la filosofía española. Filosofía cristiana de los si- 
scienze di Raimundo Lullo» ${ }^{18}$, Llull, en varias obras suyas, reivindica la instauración de los fundamentos de una nueva ciencia a partir de sus propios esquemas explicativos. De esta suerte tenemos los siguientes libros «nuevos»:

- el Tractatus novus de astronomia (10/1297, París),

- el Liber de geometria nova et compendiosa (7/1299, París),

- la Rhetorica nova (9/1301, Chipre),

- la Logica nova (5/1303, Génova),

- el Liber de novis fallaciis (10/1308, Montpellier),

- la Metaphysica nova et compendiosa (1/1310, París),

- el Liber novus physicorum et compendiosus (2/1310, París),

- el Liber de novo modo demonstrandi (9/1312, Mallorca).

Todas estas obras trabajan los distintos temas de la tradición filosóficocientífica desde el enfoque luliano. Ese carácter novedoso del $L A R$ sería coherente también con una de las autoreferencias que Llull hace de esta misma obra en el Liber de potentia, obiecto et actu: «Anima rationalis creata est et non generata, et est immortalis. Et haec in suo libro probavimus, quem de ipsa noviter fecimus» ${ }^{19}$. El hecho de que esta obra se presente con el título de Novell (en catalán) o Liber novus de anima rationali supone una voluntad de continuidad en la tradición aristotélica (tan presente a partir del siglo XII) pero a la vez una ruptura con ella, un nuevo modelo de ciencia psicológica según los parámetros del Arte.

\subsection{Localización y datación de la obra}

El libro, según se describe en los colofones, fue terminado durante la estancia de Ramon Llull en Roma, en uno de sus viajes a esta ciudad. ${ }^{20}$ En cuan-

glos XIII al XV, 2 vols., Asociación Española para el Progreso de las Ciencias, Madrid, 1939-1943 (reeditado como Carreras i Artau, T. y J., Història de la filosofia espanyola, 2 vols, Institut d'Estudis Catalans/Diputació de Girona, Barcelona/Girona, 2001), vol. 1, p. 293.

${ }^{18} \mathrm{El}$ artículo de Michela Pereira se publicó en las Actas del V Congreso Internacional de Filosofía Medieval II, Editora Nacional, Madrid, 1979, pp. 1083-1089.

19 Véase la reciente edición realizada por Núria Gómez Llauger, Ramon Llull, Liber de potentia, obiecto et actu, edición crítica, estudio introductorio y traducción de Núria Gómez Llauger, tesis dirigida por P. Villalba Varneda, Departament de Ciències de l'Antiguitat i de l'Edat Mitjana, Universitat Autònoma de Barcelona, Bellaterra, 2009. Cita de p. 88.

${ }^{20}$ Véase, por ejemplo, el colofón del manuscrito de Palma, Biblioteca Pública, 1007, f. 35ra: «Finitus fuit hic liber in Roma civitate anno incarnationis domini nostri Iesu Christi MCCXCIV, liber in quo est significata essentia animae rationalis, sua natura, suae potentiae et sui actus intrinseci et extrinseci, unde cum anima sit una pars hominis et melior pars, idcirco iste liber est multum utilis homini ad sciendum. Sciatur ergo hic liber per hominem ut per ipsum de se ipso cognitionem obtineat seu habeat <et> per cognitionem sui ipsius sciat melius intelligere, recolere et Deum amare». 
to a la fecha de redacción, ésta se presenta menos unívoca: en la mayoría de los manuscritos aparece 1294, en algún caso $1293^{21}$, años en los que no consta que estuviese en Roma. Finalmente, como acabamos de ver, aparece citado en otra obra luliana, el Liber de potentia, obiecto et actu, a la que se le ha atribuído fecha de 1296; este año sería, por tanto, el terminus ante quem. Por otro lado, el hecho de que la obra cite otras obras como el Ars inventiva veritatis, Tabula generalis, Liber de affatu, Arbor scientiae y Liber de articulis fidei, éstas dos últimas obras datadas en 1296, hace que se dé 1296 como la fecha más proba$\mathrm{ble}^{22}$.

Según lo que conocemos de la cronología de los viajes de Llull se deduce, pues, que Llull elaboró esta obra durante su estancia en Roma cuando realizó su visita al Papa Bonifacio VIII; sin embargo, como ya afirmó Hillgarth, es una datación que no alberga carácter de certeza ${ }^{23}$ aunque sí resulta probable, teniendo en cuenta los motivos aludidos y que el libro trata su objeto de estudio desde los planteamientos de la Tabula generalis, datada algo antes, en 1294.

\section{EL LAR Y AVERROES}

\subsection{La referencia a Averroes}

La obra, como hemos comentado, trata de ofrecer, a través de un innovador sistema de acercamiento filosófico, nuevas soluciones a la cuestión aristotélica de los atributos y funciones del alma racional. Aristóteles es, según vemos, la autoridad de referencia. Es conocido, sin embargo, que la traducción y recepción de las obras de Aristóteles en el mundo latino fue un fenómeno complejo: las traducciones vinieron complementadas, en muchos casos, de comentarios árabes. El caso que nos ocupa, la obra De anima aristotélica, no es en absoluto una excepción, sino uno de sus más ilustres ejemplos: Abū-l-Walīd ibn Rušd, Averroes, el comentarista árabe más importante, mostró un gran interés en la psicología, llegando a comentar hasta tres veces la obra aristotélica ${ }^{24}$.

Entre la cuestión aristotélica y la resolución luliana se imponía, así pues, un tercer elemento de plena actualidad en el universo docto del siglo XIII: la interpretación del gran Comentador a la obra aristotélica, que ya había sido versada al $34 \mathrm{ra}$.

${ }^{21}$ Cf., por ejemplo, el manuscrito de San Candido, Biblioteca della Collegiata, VIII, B. 8, f.

${ }^{22}$ Antoni Joan Pons ya apuntó la posibilidad de una confusión paleográfica entre MCCXCIV y MCCXCVI. Véase, Ramon Llull, Llibre dels articles de la fe - Llibre què deu hom creure de Déu - Llibre contra Anticrist, edición de Antoni Joan Pons, Jordi Gayà y Gret Schib (NEORL III), Patronat Ramon Llull, Palma, 1996, p. 5, n. 17.

${ }_{23}$ Al respecto véase la nota 313 en Hillgarth, Ramon Lull and Lullism ..., p. 122.

${ }^{24}$ Se trata del Compendio del libro Sobre el alma de Aristóteles, el Comentario Medio al libro Sobre el alma de Aristóteles y el Gran Comentario al libro Sobre el alma de Aristóteles. Para una antología castellana de esos textos, véase Ibn Rušd, Sobre el intelecto, edición e introducción de A. Martínez Lorca, Editorial Trotta, Madrid, 2004. 
latín en la primera mitad del siglo XIII por Miguel Escoto ${ }^{25}$. Fernand van Steenberghen, entre otros, analizó la llegada y la gran repercusión que tendrá el pensamiento del filósofo árabe en el mundo latino durante el siglo XIII ${ }^{26}$. En la Universidad de París, centro intelectual de la cristiandad, los comentarios de Averroes acompañaban la ciencia aristotélica, constituyendo la interpretación de referencia. Llull se encontró de este modo, en sus sucesivos viajes a la Universidad de París, con el establecimiento de unos planteamientos alejados e incluso contrarios a la verdad cristiana tal y como él la concebía, que significaban la implantación del pensamiento pagano en el seno de la cristiandad o, como señaló Ernest Renan, «l'islamisme en philosophie» ${ }^{27}$.

A pesar de los sucesivos avisos y prohibiciones por parte de la ortodoxia cristiana, como la condena promulgada por Étienne Tempier ${ }^{28}$, y las críticas de figuras tales como Alberto Magno y Tomás de Aquino ${ }^{29}$, el pensamiento de Averroes gozaba desde 1260 en la Facultad de Artes — con Sigerio de Brabante como mayor representante - de gran repercusión, y a partir de 1309 vive su mayor resplandor con uno de los más importantes averroístas, Juan de Jandun, entre otros. Los así llamados averroístas se caracterizaron por mantener una postura controvertida ante la relación de la fe y la filosofía, que presentaban, como mínimo, como divergentes. No es extraño, por tanto, que Llull viese en estas ideas un enemigo a combatir. Lo cierto es que la lucha contra el averroísmo no es una cuestión que se pueda desgajar de los intereses apologéticos que Llull había albergado toda su vida. Como bien señalaron Carreras i Artau ${ }^{30}$, la polémica adopta, al dirigirse a la universidad, un tono específicamente filosófico, pero el fondo es coherente con el propósito de convencer al infiel contrario a la verdad cristiana, una verdad que tal y como Llull la concibe es demostrable a través de argumentos necesarios. Sus correligionarios de París manifiestaban, así pues, una desviación de ideas que Llull no pudo considerar más que perversa e inacepta-

25 Véase la edición de IBN RUŠD, Averrois Cordubensis commentarium magnum in Aristotelis De anima libros, edición F. Stuart Crawford, Medieval Academy of America, Cambridge (Mass.), 1953. Sobre la atribución de ésta y otras traducciones de Averroes a Miguel Escoto, remitimos al riguroso análisis lingüístico que fue presentado recientemente por Dag N. Hasse, «Latin Averroes Translations of the First Half of the Thirteenth Century», en el «XII International Congress of Medieval Philosophy, Palermo, 16-22 September 2007», cuyas actas se editan por la Officina di Studi Medievali de Palermo.

${ }^{26}$ Steenberghen, en el capítulo «Invasion de la philosophie païenne» (Steenberghen F., La philosophie au XIII e siècle, Publications Universitaires, Louvain, 1966), analiza la recepción en el mundo latino del pensamiento de Aristóteles y de Averroes.

${ }^{27}$ Renan, E., Averroès et l' Averroïsme, Michel Lévy Frères Libraires Éditeurs, Paris, 1866 (reeditado como Renan, E., Averroès et l'Averroïsme, Institut für Geschichte der Arabisch-Islamischen Wissenschaften an der Johann Wolgang Goethe-Universität, Frankfurt am Main, 1985), p. 255.

${ }^{28}$ Étienne Tempier, obispo de París, publica en marzo de 1277 una lista con errores condenados por la Iglesia. Sobre este tema, véase la obra de Hissette, R., Enquête sur les 219 articles condamnés à Paris le 7 mars 1277, Publications Universitaires, Louvain, 1977.

${ }_{29}$ Ya Alberto Magno redacta un tratado De unitate intellectus contra Averroem y Tomás de Aquino discute en varias obras teorías averroístas, sobre todo la teoría del intelecto único; a dicha refutación le dedica su obra De unitate intellectus, adversus Averroistas.

${ }^{30}$ Carreras y Artau, Historia de la filosofía española..., vol. 1, p. 525. 
ble; significaban, como constata Ruedi Imbach, la negación misma de su pensamiento ${ }^{31}$.

Además de la cuestión de la irracionalidad del dogma cristiano, los averroístas eran conocidos por un aspecto muy polémico para la Iglesia y que es precisamente el que aquí nos ocupa: la doctrina del intelecto único. Tomás de Aquino fue el primero en hacer uso del término «averroístas» designando así, en su De unitate intellectus, adversus Averroistas (1270) precisamente a aquéllos que defendían la doctrina del monopsiquismo. El monopsiquismo era una de las ideas que se atribuían a Averroes más problemáticas y más controvertidas por el cristianismo, y es en relación a este tema que encontramos la referencia a Averroes en el $L A R$. El fragmento es el siguiente:

Anima non est de alia anima, quia si esset de alia anima non esset de suis principiis primis de quibus est, ut probavimus, nec non esset a generante et non a creante et nos probavimus ipsam esse a creante, et ideo dixit male Averrois in hoc quod dixit quod omnes animae sunt de una, quod est impossibile secundum quod superius probavimus in hoc quod anima est de suis primis principiis et secundariis, et de suamet essentia et non de alia, et quod est a creante et non a generante ${ }^{32}$

La referencia aparece en la tercera parte de la obra, dedicada a definir de qué es el alma y si el alma es de otro materialmente. Llull cita a Averroes en el contexto de la discusión entre creacionismo y traducianismo, esto es, de si el alma es creada directamente por Dios o si se transmite por los progenitores. Defendiendo el creacionismo, Llull asocia la posición traducianista al monopsiquismo de Averroes, en tanto que éste reduciría las almas individuales a una sola alma de la cual derivarían. Para su refutación, Llull remite, entre otros, a los principios que constituyen el alma (como la bondad, magnitud, etc.) y sus correlativos ${ }^{33}$.

${ }^{31}$ Imbach, R., «Lulle face aux Averroïstes parisiens», Raymond Lulle et le Pays d'Oc, Cahiers de Fanjeaux 22, Toulouse, 1987, pp. 270-271.

${ }^{32}$ Ms. Palma, Biblioteca Pública, 1007, f. 14va. La versión catalana dice así: «Anima no és de altra ànima, car si era de altra ànima, no seria de sos començamens primers dels quals és, segons que ja havem provat; e encara, que seria a generant e no ha crehant, e nos havem provat que és a creant. E per aço dix mal Alvar Ruy en ço que dix que totes ànimes són de una, la qual cosa és impossible, segons que demunt provat havem en ço que havem provat que ànima és de sos primers començamens e de sos secundaris e de sa matexa essència e no $d$ altra, e que és a creant e no a generant». (Ramon Llull, Libre de ànima racional, edición de Miquel Tous Gayà (ORL XXI), Comissió Editora Lul-liana, Palma, pp. 163-304 (p. 218)).

${ }^{33}$ Llull parece referirse al capítulo «De secunda specie quae est secundae partis» y en particular a la Cuestión: «Anima quid habet in se?», donde se discute la constitución del alma a partir de los principios sustanciales y accidentales y de sus correlatvios. Ms. Palma, Biblioteca Pública, 1007, f. 7vb-8ra: «Anima habet in se sua propria et naturalia principia, scilicet bonitatem, magnitudinem, durationem, potestatem, sapientiam, voluntatem, virtutem, veritatem, delectationem, differentiam, concordantiam, principium, medium, finem, maioritatem, aequalitatem et minoritatem. Et per ista principia substantialia, ex quibus constituta est, habet rationes, sicut per bonitatem habet rationem ad bonum producendum, scilicet morale bonum productum, et per magnitudinem habet rationem ad magnum bonum producendum et sic de aliis rationibus. Nec non habet anima in se ratione suae bo- 
Éste es el modo, pues, con el que Llull aborda la doctrina del intelecto único de Averroes en el $L A R$.

\subsection{La campaña antiaverroísta de Ramon Llull}

La constatación de esta referencia es notable ya que, como señaló Riedlinger, Llull pone nombre a su adversario muy tardíamente, con la Disputatio Raimundi et Averroistae de 1310, a través de la mención del término «averroístas» ${ }^{34}$. Sin embargo, se ha venido considerando ${ }^{35}$ que el antiaverroísmo de Llull comienza antes: tenemos una veintena de obras que se incluyen en la etapa antiaverroísta de Llull a partir del segundo viaje a París y la redacción de su obra Declaratio Raimundi ${ }^{36}$. Esta obra, datada en 1298, en la que se comentan los 219 artículos condenados en París en 1277 por el obispo Étienne Tempier, discute ideas averroístas aunque, como constataron Steenberghen y Cécile Bonmariage entre otros, en ningún momento de la obra aparece el término «averroísta» ni «Averroes» para nombrar la doctrina que se rebate ${ }^{37}$. Steenberghen, siguiendo este argumento, considera que no se trata de una obra especialmente antiaverroísta sino que tenía un adversario más general: un paganismo filosófico que Llull ya apreció en su segundo viaje a la Universidad de París.

Se desprende de éste y otros estudios sobre el antiaverroísmo latino que hay discrepancias en cuanto a considerar la Declaratio como obra antiaverroísta: su fecha temprana, pero sobre todo su falta de referencia concreta a Averroes o el averroísmo, permiten a autores como Steenberghen dejarla fuera de este periodo luliano.

Volviendo a nuestra obra y más allá de todo este debate, el antiaverroísmo luliano se ha vinculado tradicionalmente con la segunda y, sobre todo, la tercera estancia de Llull en París. La referencia que aparece en el $L A R$, sin embargo, surge — acogiéndonos a la datación atribuída— antes. Siendo así, ¿qué grado de

nitatis essentiale bonificativum, bonificabile et bonificare, et ratione magnitudinis essentiale magnificativum, magnificabile et magnificare, et sic de similibus [...]. Habet anima in se propria et essentialia accidentia spiritualia, scilicet quantitatem, qualitatem, relationem et alia IX praedicamentorum».

${ }^{34}$ Riedlinger, H., «Introductio generalis...», p. 33: «Id quidem mirum apud Raimundum nomen Averroistae sera tantum inveniri aetate. Neque enim Averroistae neque ipse Averroes nominantur in celebri Declaratione Raimundi, anno 1298 composita [...] Et videtur profecto, quantum quid huiusmodi de opere tantae molis magnam partem inedito asseri possit, nomen Averroistae primo apparere in Disputatione Raimundi et Averroistae, anno 1310 exeunte vel anno 1311 composita».

${ }^{35}$ Longpré, «Lulle, Raymond...», col. 1106. Riedlinger también la incluye dentro del fenómeno antiaverroísta («Introductio generalis...», p. 33).

${ }^{36}$ Ramon Llull, Declaratio Raimundi, per modum dialogi edita contra aliquorum philosophorum et eorum sequacium opiniones erroneas et damnatas a venerabili patre domino episcopo parisiensi, ediderunt M. Pereira et Th. Pindl-Büchel (ROL XVII), Brepols, Turnhout, 1989.

37 Steenberghen F., La philosophie au XIII ${ }^{e}$ siècle.., p. 511; Bonmariage, C.; Lambert, M. (trads.), Lulle et la condamnnation de 1277, Éditions Peeters, Louvain/Paris, 2006, introd., p. XIV. 
conocimiento podía tener Llull del currículum aristotélico y de la doctrina de Averroes? Respecto al grado de familiaridad que pudo tener con las obras de Averroes, la cuestión no está clara. Podemos suponer que ya en su primer viaje a París, entre 1288 y 1289, le llegó alguna noticia de los planteamientos de las tesis averroístas que se enseñaban en la Facultad de Artes; sin embargo, es sobre todo a partir de su segunda estancia que adopta una postura polemista hacia todas esas doctrinas que encuentra inadmisibles. Carreras y $\mathrm{Artau}^{38}$ consideraron que Llull no tuvo un contacto directo con las obras de Averroes, y que su conocimiento se nutría de la lista de la Condena de 1277, así como de los resúmenes que se recogían en las escuelas franciscanas, de ahí la vaguedad de sus referencias. Ruedi Imbach ${ }^{39}$ y Felipe Moreno ${ }^{40}$, defienden que esto fue así más o menos hasta finales de 1310, cuando llegó a sus manos la que fuera considerada obra luliana, el opúsculo De erroribus Averrois et Aristotelis, donde se da una lista sistemática de afirmaciones de los filósofos con mención a las obras de referencia. Sería ésta por tanto, la fuente más precisa de la que se serviría Llull en las obras a partir de finales de 1310 .

\subsection{La cuestión del monopsiquismo}

El combate de Llull contra las doctrinas de los averroístas era múltiple, si bien por encima de todo estaba una cuestión absolutamente contraria a la concepción luliana: la disociación de contenidos de fe con los contenidos de la filosofía. Llull estaba convencido de la demostrabilidad racional de los dogmas cristianos y de la concordancia entre la filosofía verdadera y la fe católica y no podía dejar de combatir la disociación de ambas. Más allá de este error, Llull se enfrenta discursivamente a otras doctrinas filosóficas defendidas por los averroístas, principalmente a la teoría sobre la ciencia divina, la potencia infinita de Dios, la eternidad del mundo y finalmente la idea del monopsiquismo, a la que alude en el $L A R$. La tan controvertida idea del intelecto único atribuída a Averroes emana sobre todo de los comentarios al tercer libro del De anima, donde Averroes postula (cap. 5 y 36) la idea de la existencia separada del intelecto material, eterno y común a todos los hombres ${ }^{41}$ :

Intellectus materialis est unus in numero in omnibus individuis hominum, non generabilis neque corruptibilis. ${ }^{42}$

\footnotetext{
${ }^{38}$ Carreras y Artau, Historia de la filosofía española...., p. 531.

39 Imbach, «Lulle face aux...», pp. 273-274.

${ }^{40}$ Moreno, F., La lucha de Ramon Llull contra el averroísmo entre los años 1309-1311, tesis doctoral dirigida por E. Rodríguez Navarro, Universidad Complutense de Madrid, Madrid, 1981, p. 128.

${ }^{41}$ La cuestión sobre la verdadera teoría del intelecto de Averroes y su interpretación es un tema complejo. En relación a este tema tenemos el estudio ya clásico de Davidson, H. A., Alfarabi, Avicenna, and Averroes on Intellect. Their Cosmologies, Theories of the Active Intellect, and Theories of Human Intellect, Oxford University Press, Oxford, 1992, pp. 220-356.

${ }^{42}$ Averrois Cordubensis commentarium magnum in Aristotelis De anima libros..., p. 401 (en la antología de A. Martínez Lorca, p. 131).
} 
A este respecto, Sigerio de Brabante es considerado uno de los averroístas latinos más importantes de la época, y defiende de manera inequívoca, como vemos, esta idea del intelecto único:

Quod sit unus intellectus in omnibus videtur. Nulla forma immaterialis, una in specie, est multiplicata secundum numerum. Sed intellectus est forma immaterialis, una in specie. Ergo non multa in numero ${ }^{43}$.

De hecho, el monopsiquismo fue considerado una de las tesis fundamentales y más características del averroísmo. Llull rechazará la idea en en varios momentos de su producción, discutiéndola más extensamente especialmente en cuatro de sus obras:

- Declaratio Raimundi, per modum dialogi edita contra aliquorum philosophorum et eorum sequacium opiniones erroneas et damnatas a venerabili patre domino episcopo parisiensi (ROL XVII, pp. 294-295 [cap. 27]; pp. 298-300 [cap. 32]), París, 1298. En esta obra que, como hemos dicho, argumenta en contra de las tesis condenadas por Tempier, el tema del monopsiquismo aparece formulado en los capítulos 27 y 32. En el capítulo 27 Llull rebate la imposibilidad de Dios de producir una pluralidad de almas: «Quod Deus non posset facere plures animas in numero». Para Llull, la sustancialidad simple de Dios no niega su posibilidad de producir la multiplicidad, del mismo modo que él es uno y también amabilidad e intelibigilidad. En el capítulo 32, bajo el lema averroísta de «Quod intellectus est unus numero omnium; licet enim ab hoc corpore separetur, non tamen ab omni», Llull retoma el tema del monopsiquismo pues al afirmar que sólo hay un intelecto, se niega la pervivencia del intelecto propio y, con ello, la posibilidad de la resurrección; además, también se niega la libertad humana, ya que se impone la calidad moral universal y se niega la capacidad del hombre de elegir el bien o el mal individualmente.

- Ars mystica theologiae et philosophiae (ROL V, pp. 420-422), París, 1309. En esta obra rebate el argumento que defiende el monopsiquismo remitiendo a la definición de los principios del Arte y a su interdependencia (la «tertia circulatio»). Además, se refiere a las pruebas dadas a favor de la verdad de la resurrección de las almas, de la creación del mundo y de la inteligibilidad de lo universal por parte de las almas particulares. Llull considera haber demostrado de manera satisfactoria con toda su argumentación los artículos de la fe racionalmente a través de la aplicación de su Arte, en contra, como dice, de aquellos «qui asserunt articulos probari non posse»(p. 422).

- Liber de perversione entis removenda (ROL V, pp. 480-482), París, 1309. Aquí da cinco argumentos en contra de la tesis monopsiquista: el $1 .^{\circ}$ y $2 .^{\circ}$ relacionados con las dignidades divinas, el $3 .^{\circ}$ a través de la doctrina de los correlativos, el $4 .^{\circ}$ y $5 .^{\circ}$ negando la eternidad del intelecto humano.

${ }^{43}$ Siger de Brabant, Quaestiones in tertium de anima, De anima intellectiva, De aeternitate mundi, edición crítica elaborada por Bernardo Bazán, Publications Universitaires, Louvain, 1972, pp. 25-26. 
- Liber reprobationis aliquorum errorum Averrois (ROL VI, pp. 305310), París, 1310. Combate de nuevo el argumento epistemológico de la imposibilidad de aprehender lo universal por parte del alma particular; defiende la multiplicidad a través del argumento de los correlativos y también a través de la misma lógica de las dignidades que requiere la multiplicidad de los intelectos.

En todas estas obras se dan razones, como vemos, que rebaten dicha doctrina: el monopsiquismo conllevaba implicaciones metafísicas que lo hacían de difícil aceptación por parte de los defensores de los dogmas de la fe cristiana. En efecto, Llull veía en la afirmación filosófica de la unidad del entendimiento y su eternidad la negación del acto creador de Dios, de la libertad humana y de la resurrección, y combate el monopsiquismo sobre todo a partir de dos frentes básicos coherentes con su cosmovisión: la teoría de las dignidades y la de los correlativos. Éstas doctrinas lulianas, como hemos visto, no sólo son centrales para la argumentación del Liber de perversione entis removenda (1309) y del Liber reprobationis aliquorum errorum Averrois (1310) sino que también caracterizan la discusión que ofrece el $L A R$.

\section{CONCLUSIONES}

¿Qué reflexiones cabe hacer tras constatar estos aspectos que se muestran en la lectura de la obra?

El tema de la citación de Averroes vincula esta obra a otras de épocas posteriores. Como hemos comentado, se percibe una evolución en el modo en que Llull se refiere a los defensores de las doctrinas consideradas averroístas, que empieza con designaciones vagas y que se va concretizando más y más a lo largo de la producción comprendida entre 1309 y 1311, sobre todo a finales de 1310.

Según se muestra en el recorrido expuesto, Llull ya en el $L A R$ discute una de las ideas averroístas, ¿tendría por ello el Liber novus de anima rationali que constituir un hito en la cronología de la cruzada antiaverroísta de Llull? La época antiaverroísta se sitúa claramente entre 1309 y 1311, y fuera quedaría como única excepción considerada hasta ahora la Declaratio Raimundi, de 1298, obra en la que no se encuentran referencias ni a Averroes ni al averroísmo; de hecho éstas vendrán bastante después, a partir de 1310. La problemática del $L A R$, con su referencia explícita a Averroes, remite, por tanto, a fechas más tardías que la que ofrece la obra. Una posibilidad que se plantea a partir de esta situación es la de la reformulación de la propia fecha de la obra: como ya hemos señalado, la fecha propuesta, 1296, parece probable, pero no indudable: el término ante quem que constituye el Liber de potentia, obiecto et actu, tampoco tiene datación del todo segura, hecho que permitiría sugerir la hipótesis de una fecha más tardía de redacción.

A todo esto, cabe añadir otro elemento que avalaría la hipótesis de una fecha posterior: hemos señalado que el libro conecta con el propósito de Llull de reformular las ciencias; en un principio, lo hace con ciencias relacionadas con el currículum del trivium y el quadrivium, sus obras de lógica, de astronomía, 
etc., serían resultado de este propósito. A partir de cierto momento, sin embargo, Llull dirige su atención hacia temas del currículum aristotélico: la Metaphysica nova y el Liber novus physicorum son expresión de este interés (véase arriba 1.2.). Esta tendencia no se manifiesta más que tardíamente, a partir de 1310; sería lógico, pues, que la redacción del $L A R$ se efectuase en torno a estos años, y no durante los años 90.

Estos dos elementos, en definitiva, remiten a fechas posteriores, momentos en los que Ramon Llull tiene un contacto más cercano con la universidad y con el currículum de la Facultad de Artes: Aristóteles y los averroístas estaban vivos en el centro del conocimiento de la cristiandad, la Universidad de París. Por el momento, esta hipótesis no puede ser más que eso, a causa de carecer de más datos que permitan modificar la cronología tradicional y dar al $L A R$ una datación más tardía.

Como vemos, lo que es en el LAR una vaga y breve referencia al monopsiquismo en otras obras se desarrolla en argumentaciones de mayor calibre llevando a cabo un desarrollo más extenso de la refutación filosófica. Cabe tener en cuenta que el $L A R$ no se presenta como una obra de discusión contra Aristóteles y Averroes, sino que ofrece un modelo alternativo. Las alusiones explícitas a Aristóteles - a través del título de la obra- - y a Averroes enmarcan la temática, reflejan las autoridades de referencia pero no exhiben en su planteamiento general y sistemático un propósito polémico, como sí lo hacen otras obras del periodo antiaverroísta.

Sea como sea, la referencia a Averroes en el $L A R$ es un hecho que hasta ahora ha pasado desapercibido y que, como hemos indicado, permite conjeturar nuevas líneas de interpretación en toda esta polémica que tiene que ver con la obra luliana y su relación con fuentes y autoridades de su tiempo, un campo de análisis tan díficil de resolver en referencia a Llull y su obra.

\section{BIBLIOGRAFÍA}

Artus, Walter, «Ramon Llull on the Pre-Eminence of Man in a God-Centered Universe», Antonianum, 66 (1991), pp. 140-154.

Bonmariage, Cécile; LAMBert, Michel (trads.), Lulle et la condamnnation de 1277, Éditions Peeters, Louvain/Paris, 2006.

CARreras y ARtau, Tomás y Joaquín, Historia de la filosofía española. Filosofía cristiana de los siglos XIII al XV, 2 vols., Asociación Española para el Progreso de las Ciencias, Madrid, 1939-1943. (Reeditado como CARRERAS I ARTAU, Tomàs y Joaquim, Història de la filosofia espanyola, 2 vols, Institut d'Estudis Catalans/Diputació de Girona, Barcelona/Girona, 2001).

DAVIDSON, Herbert A., Alfarabi, Avicenna, and Averroes on Intellect. Their Cosmologies, Theories of the Active Intellect, and Theories of Human Intellect, Oxford University Press, Oxford, 1992.

DomíngueZ, Fernando, «Works», en Alexander Fidora y Josep E. Rubio (eds.), Raimundus Lullus: An Introduction to his Life, Works and Thought (ROL - Supplementum Lullianum II), Brepols, Turnhout, 2008, pp. 125-242. 
FidORA, Alexander, «La doble tradició de les obres lul-lianes i el problema de les edicions i traduccions modernes», en Alexander Fidora y Eliseu Trenc (eds.), Segon col-loqui europeu d' estudis catalans, vol. 1: La recepció de la literatura catalana medieval a Europa, Éditions de la Tour Gile, Péronnas, 2007, pp. 5-15.

GAYÀ, Jordi, La teoría luliana de los correlativos. Historia de su formación conceptual, Impresos Lope, Palma, 1979.

Hillgarth, Jocelyn N., Ramon Lull and Lullism in Fourteenth-Century France, The Clarendon Press, Oxford, 1971.

Hissette, Roland, Enquête sur les 219 articles condamnés à Paris le 7 mars 1277, Publications Universitaires, Louvain, 1977.

IBN RUŠD, Abū-1-Walīd, Averrois Cordubensis commentarium magnum in Aristotelis De anima libros, edición F. Stuart Crawford, Medieval Academy of America, Cambridge (Mass.), 1953.

- Sobre el intelecto, edición e introducción de Andrés Martínez Lorca, Editorial Trotta, Madrid, 2004.

IMBACH, Ruedi, «Lulle face aux Averrö̈stes parisiens», Raymond Lulle et le Pays d' Oc, Cahiers de Fanjeaux 22, Toulouse, 1987, pp. 262-282.

LONGPRÉ, Éphrem, «Lulle, Raymond (Le bienheureux)», Dictionnaire de théologie catholique, vol. IX/I, Letouzey et Ané, Paris, 1926, cols. 1072-1141.

MoREno, Felipe, La lucha de Ramon Llull contra el averroísmo entre los años 13091311, tesis doctoral dirigida por Eloy Rodríguez Navarro, Universidad Complutense de Madrid, Madrid, 1981.

PereIRA, Michela, «Le 'nuove' scienze di Raimundo Lullo», Actas del V Congreso Internacional de Filosofía Medieval II, Editora Nacional, Madrid, 1979, pp. 10831089.

PRING-Mill, Robert, El microcosmos lul.lià, Editorial Moll, Palma, 1961.

RAMON LLULL, Ars mystica theologiae et philosophiae, edidit Helmut Riedlinger (ROL V), Maioricensis Schola Lullistica, Palma, 1967, pp. 259-466.

RAMON Llull, Declaratio Raimundi, per modum dialogi edita contra aliquorum philosophorum et eorum sequacium opiniones erroneas et damnatas a venerabili patre domino episcopo parisiensi, ediderunt Michela Pereira et Theodor Pindl-Büchel (ROL XVII), Brepols, Turnhout, 1989, pp. 218-402.

—, Doctrina pueril, edición crítica de Joan Santanach i Suñol (NEORL VII), Patronat Ramon Llull, Palma, 2005.

-, Liber de perversione entis removenda, edidit Helmut Riedlinger (ROL V), Maioricensis Schola Lullistica, Palma, 1967, pp. 467-506.

-, Liber de potentia, obiecto et actu, edición crítica, estudio introductorio y traducción de Núria Gómez Llauger, tesis dirigida por Pere Villalba Varneda, Departament de Ciències de l'Antiguitat i de l'Edat Mitjana, UAB, Bellatera, 2009.

-, Liber reprobationis aliquorum errorum Averrois, quos contra Christi fidem sanctam catholicam aliqui nituntur inducere, edidit Helmut Riedlinger (ROL VI), Brepols, Turnhout, 1978, pp. 288-318.

—, Libre de ànima racional, edición de Miquel Tous Gayà (ORL XXI), Comissió Editora Lul·liana, Palma, pp. 163-304. 
- Llibre dels articles de la fe - Llibre què deu hom creure de Déu-Llibre contra Anticrist, edición de Antoni Joan Pons, Jordi Gayà y Gret Schib (NEORL III), Patronat Ramon Llull, Palma, 1996.

REnAn, Ernest, Averroès et l'Averrö̈sme, Michel Lévy Frères Libraires Éditeurs, Paris, 1866. (Reeditado como RENAN, Ernest, Averroès et l'Averroïsme, Institut für Geschichte der Arabisch-Islamischen Wissenschaften an der Johann Wolgang GoetheUniversität, Frankfurt am Main, 1985).

RIEDLINGER, Helmut, «Introductio generalis» en Raimundi Lulli Opera Latina V, Opera Parisiensia Anno MCCCIX composita, edidit Helmut Riedlinger, Maioricensis Schola Lullistica, Palma, 1967.

SigER DE BRABANT, Quaestiones in tertium de anima, De anima intellectiva, De aeternitate mundi, edición crítica elaborada por Bernardo Bazán, Publications Universitaires, Louvain, 1972.

SteEnberghen, Fernand Van, La philosophie au XIII e siècle, Publications Universitaires, Louvain, 1966.

Recursos en Internet: http://orbita.bib.ub.es/llull/index.asp 